\section{BMJ Open Respiratory Research}

\title{
How can we relieve gastrointestinal symptoms in people with cystic fibrosis? An international qualitative survey
}

\author{
Sherie Smith (D) , ${ }^{1}$ Nicola Rowbotham, ${ }^{1}$ Gwyneth Davies, ${ }^{2}$ Katie Gathercole, ${ }^{3}$ \\ Sarah J Collins, ${ }^{4}$ Zoe Elliott, ${ }^{5}$ Sophie Herbert, ${ }^{1}$ Lorna Allen, ${ }^{6}$ Christabella Ng, ${ }^{1}$ \\ Alan Smyth ${ }^{1}$
}

To cite: Smith $\mathrm{S}$,

Rowbotham N, Davies G, et al. How can we relieve gastrointestinal symptoms in people with cystic fibrosis? An international qualitative survey. BMJ Open Resp Res 2020;7:e000614. doi:10.1136/ bmjresp-2020-000614

Received 20 April 2020 Revised 22 July 2020 Accepted 10 August 2020

Check for updates

(C) Author(s) (or their employer(s)) 2020. Re-use permitted under CC BY-NC. No commercial re-use. See rights and permissions. Published by BMJ.

${ }^{1}$ Child Health Obstetrics and Gynaecology, University of Nottingham, Nottingham, UK

${ }^{2}$ Institute of Child Health, UCL, London, UK

${ }^{3}$ Person with CF, University of Leeds, Leeds, UK

${ }^{4}$ CF Department, Royal

Brompton and Harefield NHS

Trust, London, UK

${ }^{5}$ Mother of Children with CF, Nottingham, UK

${ }^{6}$ Cystic Fibrosis Trust, London, UK

Correspondence to

Sherie Smith;

sherie.smith@nottingham. ac.uk

\section{ABSTRACT}

Introduction Relieving gastrointestinal (GI) symptoms was identified as a 'top ten' priority by our James Lind Alliance Priority Setting Partnership in cystic fibrosis (CF). We conducted an online survey to find out more about the effect of Gl symptoms in CF.

Methods We co-produced an online survey distributed to the CF community via web-based platforms. The survey consisted of open and closed questions designed to help us learn more about the effects of Gl symptoms for people with CF (pwCF). We analysed the data using descriptive statistics and thematic analysis. We promoted the survey via social media and web-based platforms which allowed respondents from any country to take part. Our participants came from the CF community, including: adults and children with CF, parents and close family of pwCF and healthcare professionals (HCPs) working with pwCF. Results There were 276 respondents: 90 (33\%) pwCF, 79 (29\%) family, 107 (39\%) HCPs. The most commonly reported symptoms by lay respondents were stomach cramps/pain, bloating and a 'combination of symptoms'. The top three symptoms that HCPs said were reported to them were reduced appetite, bloating and constipation. Almost all (94\% (85/90)) HCPs thought medications helped to relieve Gl symptoms but only $58 \%(82 / 141)$ of lay respondents agreed.

Conclusions Our survey has shown that Gl symptoms among our participants are prevalent and intrude on daily lives of pwCF. There is a need for well-designed clinical studies to provide better evidence for management of GI symptoms and complications.

\section{INTRODUCTION}

Cystic fibrosis (CF) is the most common autosomal recessive inherited disease in people of European descent. ${ }^{1}$ It was once considered a disease of childhood as survival was rarely beyond the childhood years. With the advent of new treatments and novel drugs, people with CF (pwCF) are now living longer and often well into adulthood. For a baby born in 2018, the median life expectancy is now 47.3 years. ${ }^{2}$ The respiratory system is usually the

\section{Key messages}

How to relieve gastrointestinal (Gl) symptoms in cystic fibrosis (CF) was identified as a top research priority, but required further work to better understand the research question.

> We were able to identify the common symptom(s) and their impact on daily life in CF, including differences between lay and professional expectations of symptom relief.

- We hope that our findings will help inform welldesigned clinical studies to provide better evidence for management of Gl symptoms and complications in CF.

main focus of treatment as respiratory disease remains the major cause of morbidity and mortality in $\mathrm{CF}^{2}{ }^{2}$ However, $\mathrm{CF}$ is a multisystem disease which necessitates a burdensome regimen of treatments. The CF landscape is changing and problems which may have been overlooked are now becoming a priority for people living with the condition and those providing CF care.

The gastrointestinal (GI) system is commonly affected in pwCF, the majority of whom have pancreatic insufficiency, requiring pancreatic enzyme supplements with meals. ${ }^{3}$ In any 1 year, around $5 \%$ of patients will suffer the most severe GI complication, distal intestinal obstruction syndrome (DIOS). ${ }^{2}$ The prevalence of liver disease is around $13 \% .^{2} \mathrm{GI}$ symptoms which are reported in CF include: bloating, flatulence, abdominal pain and gurgling (borborygmi), with a prevalence of around $65 \%$. With improved life expectancy, symptoms which previously received little attention are now having a greater impact on the lives of those with GI symptoms. The burden of GI symptoms in pwCF is higher than the general population. For example, 
irritable bowel syndrome (characterised by abdominal pain, bloating, flatulence, diarrhoea and/or constipation) affects $10 \%-25 \%$ of the UK population, only half of whom will present to primary care. ${ }^{5}$

We recently carried out a James Lind Alliance Priority Setting Partnership in CF which resulted in a 'top ten' list of research priorities decided jointly by healthcare professionals (HCPs) and the patient community through online surveys and a final workshop. ${ }^{6}$ Some of these priorities were broad topics which did not translate readily into testable hypotheses. The priority ranked in position two was: 'How can we relieve gastro-intestinal symptoms such as stomach pain, bloating and nausea?'. In a continuation of the priority setting work and in conjunction with the CF community, we aimed to:

1. Understand the prevalence and impact of GI symptoms in CF.

2. Gather ideas for future clinical research into approaches to relieving GI symptoms.

Some results of this work have been previously published as an abstract ${ }^{7}$ and shared on social media with the CF community.

\section{METHODS}

The UK National Institute for Health Research (NIHR) supports the James Lind Alliance and agreed to us continuing our priority setting work under the branding 'James Lind CF2' to investigate some of the research questions from the original priority setting partnership in more depth. The protocol for this continuation work can be found here (also online supplemental file 1) in https:// nottingham-repository.worktribe.com/output/1195186. The Standards for Reporting Qualitative Research Reporting guidelines were used to report the methods used. $^{8}$

\section{Survey development}

A steering group, representative of the CF community (both patients/parents and HCPs), guided the production of an electronic questionnaire (SurveyMonkey). The survey (online supplemental file 2) was designed to help us understand how GI symptoms impact on the daily lives of pwCF and the measures they are already taking to help alleviate or avoid them. The survey included a mixture of closed and open questions and there was opportunity to expand in free text comment boxes. After an initial screening question to determine eligibility, the survey was divided into separate streams for HCPs and lay respondents although we aimed to elicit the same information from different perspectives. Questions related to three main themes:

1. Presence of a diagnosed GI complication (lay respondents only).

2. Symptoms (including type, frequency and severity).

3. Relief of symptoms (including medications, dietary/ exercise factors, non-medical interventions).
Data collection

The survey was promoted online via Twitter (@questionCF), professional networks, UK CF Trust, US CF Foundation and NIHR. It was an open invitation to anyone in the CF community rather than it being sent to a discreet sample of the population.

The survey was anonymous and where contact details were left for further interest in the study, these were separated from the survey data and stored in compliance with General Data Protection Regulation guidelines. Participants were asked to read and agree to our privacy statement before progressing through to the survey. It was designed to be as inclusive as possible, with no restrictions on age or location, and was open for a period of 4 weeks between June and July 2018. The University of Nottingham Research Ethics Committee deemed this programme of work not to require ethical approval.

\section{Data analysis}

The data were downloaded from SurveyMonkey to Microsoft Excel for analysis of closed questions including a description of the respondent demographics and frequency of responses.

Free text data were downloaded to NVivo V.12 for thematic analysis. This was done using an iterative process using content and thematic analysis. The word frequency function was used for questions where there was free text content to help generate themes. Linked words such as 'bloat' and 'bloating' were combined and commonly used words that did not relate directly to the results were removed (eg, 'sometimes', 'symptoms'). These themes were then applied across the whole dataset and the data were coded into these themes. Many of the comments fell into more than one theme and so were recorded separately under each theme to which the comment related. It is possible that this could lead to an over representation of some responses but it makes the distinction between the quantitative numbers of people reporting that they experience a particular symptom and the frequency with which it is mentioned across the whole dataset. The coding was validated by two authors to ensure consistency across the emergent themes and how the data were assigned to each theme (SS, NR).

\section{Patient and public involvement}

This work was driven by patient and public involvement, and this remained central to all stages of design, implementation and dissemination. The study was guided by a steering group which included two pwCF and a parent of children with CF. They were all involved in the design of the study, communication and interpretation of the results. Our lay representatives were instrumental in designing the survey to ensure it would be clear and understandable and also helped publicise the survey to the wider CF community via social media. 


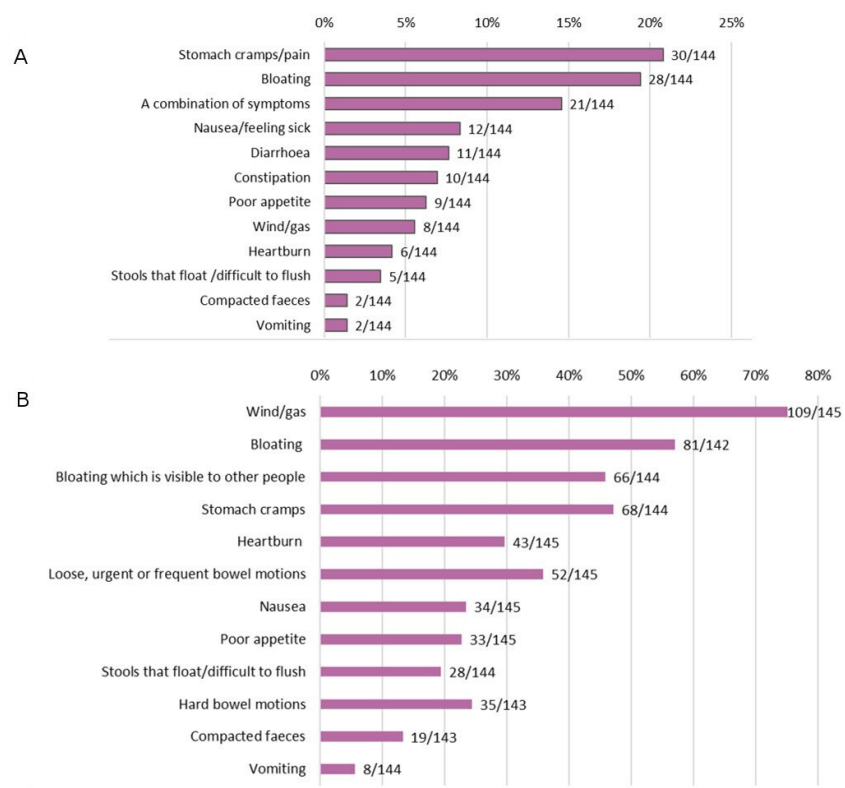

c

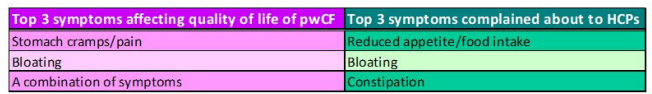

Figure 1 Gl symptoms experienced by pwCF. (A)

Symptoms which most affect quality of life for pwCF. (B)

Symptoms which are experienced every day or most days.

(C) Top three symptoms affecting quality of life for pwCF and the top three complained about to HCPs. HCPs, healthcare professionals; pwCF, people with cystic fibrosis.

\section{RESULTS}

In total, 276 survey responses were received including 90 (33\%) from pwCF; 79 (29\%) from parents, relatives or friends and $107(39 \%)$ responses from HCPs. Almost half of the HCPs $(44 \%, n=47)$ were dietitians. Median age of respondents was 37 years (range 15-74 years); median age of pwCF (including children with a parent answering on their behalf) was 12 years (range $0-74$ years). We had responses from 15 countries, with $171(62 \%)$ from the UK and $20(7 \%)$ from the USA. Not all survey participants responded to each question so, where we present numbers and percentages, we have stated the denominator. Number of respondents to each question is given in online supplemental file 3.

Diagnosed pancreatic insufficiency and GI complications of CF were reported by a large number of our lay respondents, with $84 \%(132 / 157)$ having been diagnosed

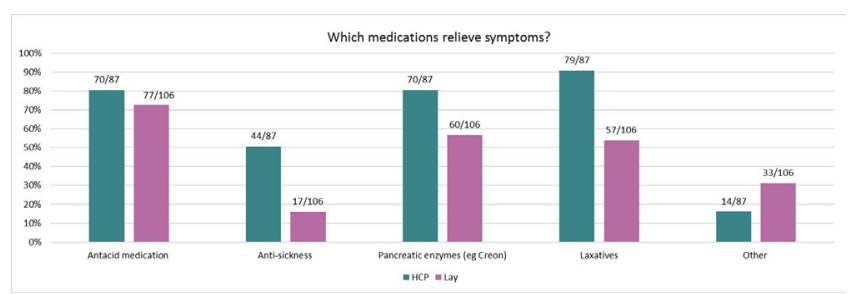

Figure 2 Relief of symptoms. HCP and lay views on which medications relieve symptoms. HCP, healthcare professional. with pancreatic insufficiency; 39\% (61/157) having had DIOS; 20\% (32/157) having CF-related liver disease (CFRLD) and 32\% (50/157) having had meconium ileus in the newborn period. Of the respondents who reported these conditions, almost half had at least two diagnoses $(48 \%, 76 / 157)$ and a small percentage reported all four diagnoses listed $(6 \%, 10 / 157)$.

All of the participants who reported pancreatic insufficiency also reported having experienced GI symptoms at some time compared with 26 out of $36(72 \%)$ pancreatic sufficient participants.

Figure 1 shows the most common symptoms affecting quality of life in pwCF (figure 1A) and the frequency with which symptoms occur (figure 1B). The most commonly reported symptoms were stomach cramps/pain, bloating and a 'combination of symptoms' (this was reported as the concomitant effect of symptoms occurring together including gas, pain, nausea, bloating, diarrhoea and vomiting). The top three symptoms that HCPs said were reported to them were reduced appetite, bloating and constipation (figure 1C). Missing school or work because of GI symptoms was reported by $66 \%(97 / 146)$ of the lay respondents and a similar proportion said that symptoms have affected their confidence or self-image $(65 \%$, 95/145). While 95\% (147/155) reported being able to discuss GI symptoms with their CF team, only $26 \%$ $(41 / 155)$ reported having the opportunity to discuss these with a gastroenterologist on their CF team.

We asked HCPs and pwCF whether they felt that medications helped to relieve GI symptoms and while $94 \%(85 / 90)$ of HCPs thought that they did, only $58 \%$ $(82 / 141)$ of lay respondents felt the same. Figure 2 shows which types of medications were reported to help the most, with the top two for HCPs being laxatives and antacid medication and the top two for lay respondents being antacid medication and pancreatic enzymes. Only half of lay respondents felt that laxatives helped to relieve symptoms. We also asked if any non-medical interventions, such as diet and exercise, helped relieve symptoms. Just under half of our lay respondents reported that symptoms were relieved by non-medical interventions $(46 \%, 58 / 126)$. Of those respondents who answered this question, $28 \%(35 / 126)$ felt that diet helped and $18 \%$ (23/126) thought that exercise improved symptoms. A higher proportion of HCPs felt that a change of diet helped to relieve symptoms $(52 \%, 46 / 88)$. Other methods used by pwCF to relieve GI symptoms included massage, heat, probiotics, keeping hydrated and avoiding triggers such as over-eating.

\section{Thematic analysis}

We identified recurrent themes during the analysis of the free text data. The themes mentioned most frequently (figure 3) focused on physical effects such as pain, discomfort and bloating, and how this impacts on everyday life.

I cannot go to work when I have stomach pain and cramps as the pain makes it impossible to concentrate, 


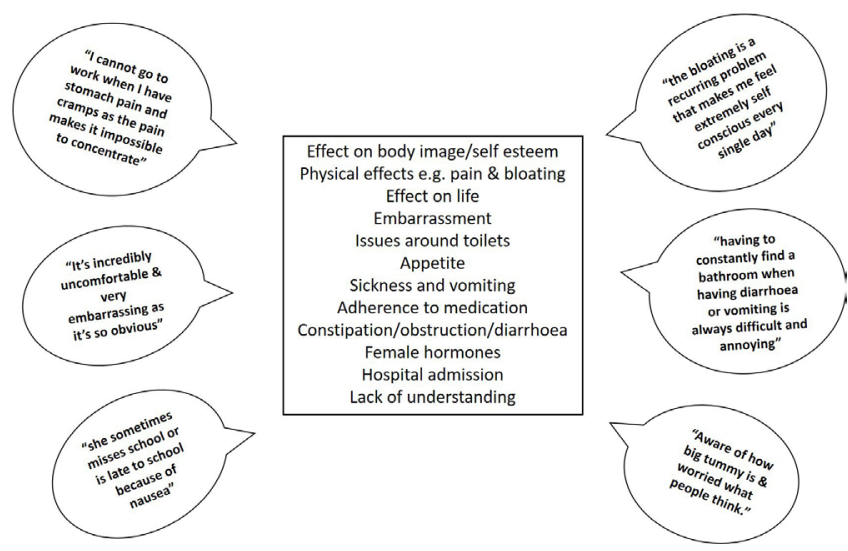

Figure 3 Themes emerging from the free text responses and direct quotes relating to the impact of Gl symptoms. GI, gastrointestinal.

in general I get the stomach pain and cramps when I have diarrhoea so I wouldn't want to go to work as it's a shared toilet and would feel self-conscious that I was up and down from my desk to go to the toilet. It's embarassing. (pwCF, UK)

If I'm cramping then I can't work or exercise. (pwCF, Canada)

Constant stomach pain, enormous bloating, unpredictable bowel movements-it has been debilitating for my daughter, who can't often exercise properly, which is bad for her chest and general health and wellbeing. She often misses social events. (Parent of a child with CF, England)

Bloating to the extent that pwCF felt as though they 'looked pregnant' and being unable to wear clothes of

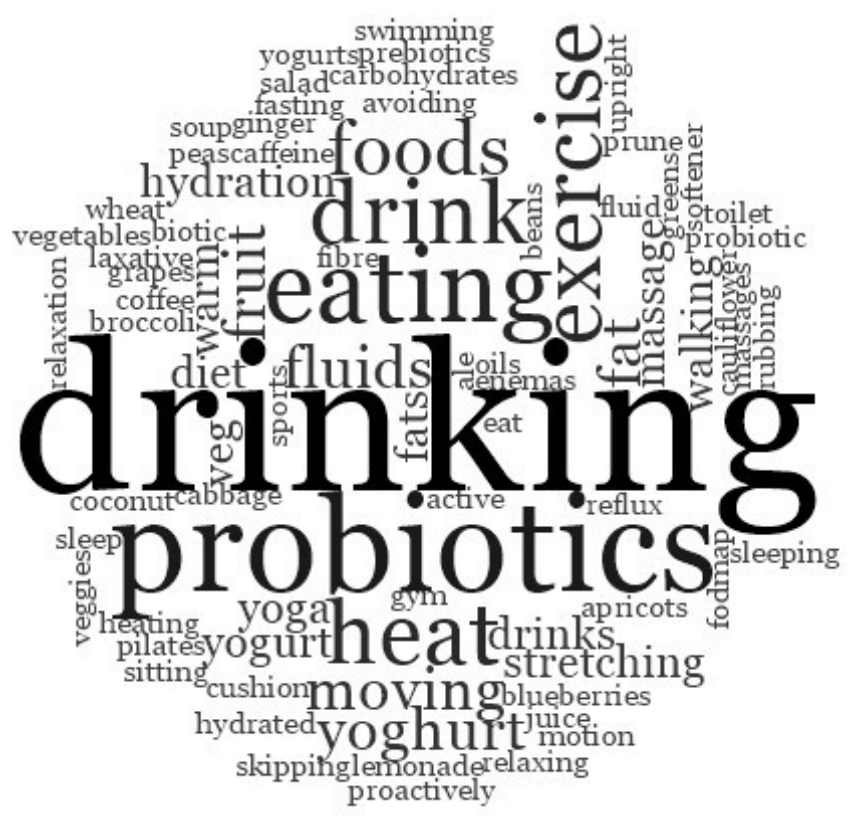

Figure 4 Word cloud showing other interventions which pwCF said helped to relieve symptoms. pwCF, people with cystic fibrosis. their choice was mentioned frequently along with the concomitant effect on self-image and self-esteem.

I now have to buy clothes with my bloated stomach in mind. I feel self-conscious when I am bloated. I try to hide my bloating from others. (pwCF, UK)

Feeling bloated a lot of the time and therefore feeling uncomfortable with the way you feel and look. Having to choose your clothing so it doesn't show your bloated stomach. (pwCF, UK)

Embarrassing when you have gas and self-conscious about my body when I have a distended stomach. (pwCF, Canada)

We also asked our respondents what measures they found helped control GI symptoms. Responses included medications that helped, along with non-pharmaceutical interventions such as diet, exercise and alternative therapies. A wide range of measures were reported, shown in a frequency word cloud (figure 4).

Daily probiotics, eating more vegetables and lower refined carbohydrates has made my bloating and gas virtually normal. (pwCF, Australia)

I hold a hot water bottle on my stomach area and it relieves the pain and also seems to help get things moving. (pwCF, UK)

\section{DISCUSSION}

Our survey has shown that within our respondent group GI symptoms are prevalent, intrusive and have a daily impact on the lives of pwCF and those living and working with them. Common symptoms reported are pain, bloating and wind. There is a difference between lay respondents and HCPs with regard to whether they feel symptoms are eased by medication, with a higher proportion of HCPs feeling that medications relieved symptoms than lay respondents. Themes emerging from the qualitative analysis were largely around feelings of embarrassment, poor self-image and effect on everyday life.

Using an anonymous survey has allowed us to learn more about the impact of GI symptoms on pwCF than would be possible through interviews or focus groups. The use of a web-based platform, while necessary to prevent cross-infection from face to face contact, has meant that our survey was accessible to participants internationally and we encouraged honesty and openness. Many of the free text comments related to embarrassment and it is possible that this is a subject which pwCF do not find easy to discuss with their CF team.

Traditionally CF treatment has had a respiratory focus as respiratory failure is the most common cause of death in pwCF. $^{9}$ However, most pwCF have pancreatic insufficiency necessitating pancreatic enzyme replacement therapy ${ }^{3}$ and all pwCF, completing a CF-specific questionnaire, reported GI symptoms in the preceding 3 months. ${ }^{10}$ Tools for assessing GI symptoms in pwCF are still in development and scoring systems designed for other GI conditions are often used in CF (eg, GI Symptom Rating Scale; 
Irritable Bowel Syndrome Symptom Severity Score). ${ }^{4}$ Treatment guidelines focus on optimising nutrition, pancreatic enzyme supplements and the management of DIOS, but acknowledge the lack of high-quality evidence to guide recommendations. ${ }^{11}$ Current UK National Institute for Health and Care Excellence guidelines outline treatment regimens to maintain optimum nutritional status but there is little guidance on symptom control. ${ }^{12}$ There is imprecision in the terminology used for GI symptoms which may lead to a difference between HCPs and lay people reporting symptoms.

The study highlights the need for evidence-based treatments to alleviate distressing GI symptoms in addition to optimisation of current available treatments. It is clear that pwCF already use a range of different methods in an attempt to control symptoms including medications and non-medical interventions. A recent Cochrane review included 12 randomised controlled trials that looked at probiotics for CF but none of the trials reported on GI symptoms. ${ }^{13}$ Early work using MRI is aiming to identify physiological markers of where the CF GI system differs from that of healthy controls. ${ }^{14}$ New treatments that correct the underlying defect in CF transmembrane conductance regulator (CFTR) proteins have been shown to be effective in pwCF who have specific gene mutations. ${ }^{15}$ While most trials have measured respiratory outcomes, it is possible that these novel treatments may also correct some of the underlying mechanisms of GI problems. CFTR modulator treatment has been shown to be associated with weight gain ${ }^{16}$ while small uncontrolled studies have shown that CFTR modulators have an effect on small intestinal $\mathrm{pH},{ }^{17}$ as well as microbiota and intestinal inflammation. ${ }^{18}$ Further, well-designed studies of the effects of CFTR modulators on GI symptoms are needed.

\section{Limitations}

Our study provides a snapshot of how patients in a small sample of the CF population experience GI symptoms and how they perceive the effectiveness of treatments for these symptoms. While this gives some insight into what professional and lay priorities might be in terms of future research, it may not be representative of the whole CF population. The UK CF Registry annual data report for 2018 shows that $13.2 \%$ of all registered patients have CFRLD compared with $20 \%$ of the participants in our study reporting CFRLD. Similarly there is a lower prevalence of DIOS in the registered population compared with our study population ( $5.2 \%$ vs $39 \%$, respectively). ${ }^{2}$ Furthermore, we were unable to undertake a subanalysis by genotype or to look at the effects of common CF interventions, such as antibiotics, on symptom type and frequency as this was beyond the scope of our survey. Survey respondents are likely to be pwCF whose GI symptoms are particularly problematic. We asked a broad question: 'Are symptoms relieved by any medication?'. Our data do not allow the response of specific symptoms to specific drugs to be analysed. Many of the medications prescribed for GI symptoms do not have immediate effects which will impact on patient reporting of the perceived effectiveness of each treatment.

Although the lay and professional surveys were designed to be very similar in terms of the questions asked, they could not be identical and so our results are very useful for giving a general picture and suggesting possible similarities and differences. While we have compared the symptom frequency reported by patients to what professionals hear in consultations, we acknowledge that the two groups may not have a common language to describe symptoms.

Our work has shown that GI symptoms are often intrusive and debilitating in the daily lives of pwCF and for those affected can cause considerable discomfort and inconvenience. Many respondents talked about the embarrassment of dealing with GI symptoms and our survey has allowed pwCF to describe these anonymously, where they may find discussing it with their CF team difficult. This, along with differences in describing and reporting symptoms may explain some of the mismatch between HCP and lay responses. Our study has also highlighted the importance of less measurable outcomes such as bloating, stomach cramps and embarrassment. Future research around screening and reporting of GI symptoms in regular CF clinics would be beneficial both in terms of defining symptoms and identifying other conditions such as GI cancers. ${ }^{19}$ As treatment for CF is improving and people are living longer, there is the increased risk of developing age-related conditions which need to be distinguished from CF-related symptoms.

\section{CONCLUSIONS}

This study highlights some of the effects of GI symptoms on the lives of pwCF and identifies an area for improved communication with their CF team. The most common symptoms affecting quality of life in our study were bloating and stomach cramps. There is an unmet need for well-designed clinical studies to provide a better evidence base of management of GI symptoms and complications such as DIOS.

Acknowledgements We would like to thank all the pwCF and their families, and the multidisciplinary CF HCPs for taking part in this survey. Members of the James Lind Alliance CF2 steering group include: Brownlee K, Collins S, Daniels T, Davies G, Duff AJA, Elliot ZC, Gathercole K, Herbert S, Hurley MN, Leighton PA, Rayner OC, Rowbotham NJ, Smith SJ, Chandran S, Nash EF, Smyth AR (Chair), Wilson P.

Contributors AS and NR conceived and designed the study. SS, NR, GD, KG, SJC, ZE, SH, LA and AS were involved in the design and promotion of the survey. ZE promoted and monitored the promotion of the survey on Twitter. SS and NR performed data analysis and all authors were involved in interpretation of the analysis. CN provided expertise in the field of gastrointestinal function. SS drafted the manuscript and all authors reviewed and approved it for publication.

Funding This work was supported by the UK CF Trust (Grant number SDF002). NR was an NIHR Academic Clinical Fellow at University of Nottingham. GD was an NIHR Clinical Trials Fellow and was previously supported by a NIHR Clinical Lectureship at UCL. AS is supported by the Nottingham NIHR Biomedical Research Centre. All research at Great Ormond Street Hospital NHS Foundation Trust and UCL Great Ormond Street Institute of Child Health is made possible by the NIHR Great Ormond Street Hospital Biomedical Research Centre. 
Competing interests GD reports personal fees from Chiesi, outside the submitted work. CN reports grants from Cystic Fibrosis Foundation, grants from Cystic Fibrosis Trust, grants and other from Vertex Pharmaceuticals, outside the submitted work. NR has given lectures at meetings sponsored by TEVA and has attended meetings sponsored by Vertex. AS reports a research grant and personal fees from Vertex. He has spoken at meetings supported by Teva and Novartis. These activities are outside the submitted work. In addition, AS has a patent for 'Alkyl quinolones as biomarkers of Pseudomonas aeruginosa infection and uses thereof' issued.

Patient and public involvement Patients and/or the public were involved in the design, or conduct, or reporting, or dissemination plans of this research. Refer to the Methods section for further details.

Patient consent for publication Not required.

Ethics approval This was a continuation of our James Lind Alliance priority setting work and therefore did not require ethical approval. The survey was anonymous and promoted through socialmedia.

Provenance and peer review Not commissioned; externally peer reviewed.

Data availability statement No data are available. The survey, numbers of respondents and protocol for the James Lind CF2 project as a whole are included as supplementary documents. No additional data are available.

Open access This is an open access article distributed in accordance with the Creative Commons Attribution Non Commercial (CC BY-NC 4.0) license, which permits others to distribute, remix, adapt, build upon this work non-commercially, and license their derivative works on different terms, provided the original work is properly cited, appropriate credit is given, any changes made indicated, and the use is non-commercial. See: http://creativecommons.org/licenses/by-nc/4.0/.

ORCID iD

Sherie Smith http://orcid.org/0000-0002-4143-5364

\section{REFERENCES}

1 Farrell $\mathrm{P}$, Férec $\mathrm{C}$, Macek $\mathrm{M}$, et al. Estimating the age of p.(Phe508del) with family studies of geographically distinct European populations and the early spread of cystic fibrosis. Eur J Hum Genet 2018;26:1832-9.

2 Cystic Fibrosis Trust. UK cystic fibrosis registry annual data report 2018. London, 2019.

3 Cystic Fibrosis Genotype-Phenotype Consortium. Correlation between genotype and phenotype in patients with cystic fibrosis. $N$ Engl J Med 1993;329:1308-13.
4 Hayee Bu'Hussain, Watson K-L, Campbell S, et al. A high prevalence of chronic gastrointestinal symptoms in adults with cystic fibrosis is detected using tools already validated in other Gi disorders. United European Gastroenterol J 2019;7:881-8.

5 Williams JG, Roberts SE, Ali MF, et al. Gastroenterology services in the UK. The burden of disease, and the organisation and delivery of services for gastrointestinal and liver disorders: a review of the evidence. Gut 2007;56:1-113.

6 Rowbotham NJ, Smith S, Leighton PA, et al. The top 10 research priorities in cystic fibrosis developed by a partnership between people with CF and healthcare providers. Thorax 2018;73:388-90.

7 Smith S, Rowbotham NJ, Davies G, et al. P282 gastrointestinal symptoms in people with cystic fibrosis: a survey of lay and professional views. J Cyst Fibros 2019;18:S137.

8 O'Brien BC, Harris IB, Beckman TJ, et al. Standards for reporting qualitative research: a synthesis of recommendations. Acad Med 2014;89:1245-51.

9 Cystic Fibrosis Foundation. 2017 patient registry annual data report. Bethesda, Maryland, 2018.

10 Tabori H, Arnold C, Jaudszus A, et al. Abdominal symptoms in cystic fibrosis and their relation to genotype, history, clinical and laboratory findings. PLoS One 2017;12:e0174463.

11 UK Cystic Fibrosis Trust Nutrition Working Group. Nutritional management of cystic fibrosis. London: Cystic Fibrosis Trust, 2016.

12 NICE. Cystic fibrosis: diagnosis and management. London, 2017.

13 Coffey MJ, Garg M, Homaira N, et al. Probiotics for people with cystic fibrosis. Cochrane Database Syst Rev 2020;1:CD012949.

$14 \mathrm{Ng} \mathrm{C}$, Dellschaft NS, Hoad CL, et al. Postprandial changes in gastrointestinal function and transit in cystic fibrosis assessed by magnetic resonance imaging. J Cyst Fibros 2020. doi:10.1016/j. jcf.2020.06.004. [Epub ahead of print: 16 Jun 2020].

15 Southern KW, Patel S, Sinha IP, et al. Correctors (specific therapies for class II CFTR mutations) for cystic fibrosis. Cochrane Database Syst Rev 2018;8:CD010966.

16 Ramsey BW, Davies J, McElvaney NG, et al. A CFTR potentiator in patients with cystic fibrosis and the G551D mutation. N Engl J Med 2011;365:1663-72.

17 Gelfond D, Heltshe S, Ma C, et al. Impact of CFTR modulation on intestinal $\mathrm{pH}$, motility, and clinical outcomes in patients with cystic fibrosis and the G551D mutation. Clin Trans/ Gastroenterol 2017;8:e81.

18 Ooi CY, Syed SA, Rossi L, et al. Impact of CFTR modulation with ivacaftor on gut microbiota and intestinal inflammation. Sci Rep 2018;8:17834.

19 Yamada A, Komaki Y, Komaki F, et al. Risk of gastrointestinal cancers in patients with cystic fibrosis: a systematic review and meta-analysis. Lancet Oncol 2018;19:758-67. 